\title{
How do associative and phonemic overlap interact to boost illusory recollection?
}

\author{
Authors: Keith A. Hutchison, Michelle L. Meade, \\ Nikolas S. Williams, Krista D. Manley, \& Jaimie C. \\ McNabb
}

This is an Accepted Manuscript of an article published in Memory on October 24, 2017, available online: http://www.tandfonline.com/10.1080/09658211.2017.1393091.

Hutchison, Keith A, Michelle L Meade, Nikolas S Williams, Krista D Manley, and Jaimie C McNabb. "How do associative and phonemic overlap interact to boost illusory recollection?." Memory (October 2017): 1-8. DOI: 10.1080/09658211.2017.1393091.

Made available through Montana State University's $\underline{\text { ScholarWorks }}$ scholarworks. montana.edu 


\title{
How do associative and phonemic overlap interact to boost illusory recollection?
}

\author{
Keith A. Hutchison ${ }^{a}$, Michelle L. Meade ${ }^{a}$, Nikolas S. Williams ${ }^{b}$, Krista D. Manley ${ }^{c}$ and Jaimie C. McNabb ${ }^{d}$ \\ ${ }^{a}$ Department of Psychology, Montana State University, Bozeman, MT, USA; ${ }^{\mathrm{b}}$ Department of Psychology, Macquarie University, Sydney,

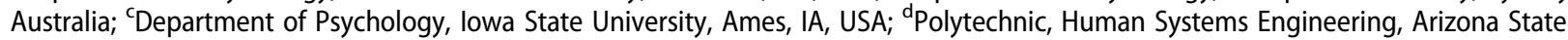 \\ University Polytechnic, Meza, AZ, USA
}

\begin{abstract}
This project investigated the underlying mechanisms that boost false remember responses when participants receive study words that are both semantically and phonologically similar to a critical lure. Participants completed a memory task in which they were presented with a list of words all associated with a critical lure. Included within the list of semantic associates was a target that was either semantically associated (e.g., yawn) to the critical lure (e.g., sleep) or shared the initial (e.g., slam) or final (e.g., beep) phoneme(s) with the critical lure. After hearing the list, participants recalled each list item and indicated whether they just knew it was on the list or if they instead recollected specific contextual details of that item's presentation. We found that inserting an initial phonemic overlap target boosted experiences of recollection, but only when semantically related associates were presented beforehand. The results are consistent with models of spoken word recognition and show that established semantic context plus initial phonemic overlap play important roles in boosting false recollection.

KEYWORDS

False recall; hybrid lists;

source monitoring
\end{abstract}

One of memory's nastiest tricks is illusory recollection. Or the detailed subjective experience that one had previously encountered an event that never occurred. (Gallo, 2006, p. 75)

Roediger and McDermott (1995) revised a paradigm created by Deese (1959) in which participants study lists of words (e.g., bed, rest, tired, night, ...) that are all associated to a non-presented critical lure (e.g., sleep). Participants in this paradigm typically recall the critical lure about as often as the actual words on the list. Moreover, these false memories are robust and powerful such that participants often report recollecting a detailed experience of having seen or heard the word during the list presentation (Payne, Elie, Blackwell, \& Neuschatz, 1996). This is a particularly nasty trick of memory indeed, because society weighs recollective accounts particularly heavily when judging a person's memory, such as during eyewitness testimony.

A particularly interesting finding by Watson and colleagues is that false memories of critical lures (e.g., sleep) are dramatically enhanced when lists contain a combination of both semantic (e.g., tired) and phonological (e.g., sleeve) associates, relative to lists comprised purely of one type (Watson, Balota, \& Roediger, 2003; Watson, Balota, \& Sergent-Marshall, 2001). For example, in Watson et al.'s (2003) Experiment 1, they visually presented participants with a list of 10 semantic associates plus 3 additional words that were either all unrelated or contained between 1 and 3 semantic or phonological associates. They found that false recall rates when adding 0,1 , 2 and 3 phonological associates were .24, .34, .41 and.49, respectively, in contrast to $.21, .24, .26$ and .23 , respectively, when adding semantic associates. Thus, adding 3 phonological associates to a list of 10 semantic associates doubled the false recall rate, whereas adding 3 additional semantic associates had little effect. In additional experiments, Watson and colleagues demonstrated that hybrid lists containing both sematic and phonological associates produced over-additive effects, such that false recall from hybrid lists was greater than the sum of phonological list false recall plus semantic list false recall. Such over-additivity occurred with young adults, older adults and individuals diagnosed with dementia of the Alzheimer's type and extended beyond recall to false recognition and even remember responses. Recently, Finley, Sungkhasettee, Roediger, and Balota (2017) tested every combination of mixing semantic and phonological associates, from all semantic items to all phonological items. They found that false recall increased as lists became more hybrid, but that the largest increase occurred with the first hybrid item (i.e., a semantic associate added to a phonological 
list or vice-versa) and that further "mixing" of the lists produced diminishing increases in false recall after including 2-3 hybrid items. Thus, just adding a couple items is sufficient to boost false recall.

Why do hybrid lists create such super-additive recall and experiences of remembering? Watson et al. (2003) offered three possible explanations. The first explanation was based on associative activation theories of false memory (Deese, 1959; Roediger, Balota, \& Watson, 2001; Underwood, 1965), which assume false memory arises primarily due to activation of the critical lure from list items. According to Watson et al., a simple associative activation account could explain the over-additive pattern if it is assumed that (1) there are distinct phonological and semantic networks (cf., Tse, Li, \& Neill, 2011) and (2) activation within each network reaches an asymptote such that adding additional associates of the same type produces diminishing returns. The result of adding additional semantic associates in their Experiment 1 supports such an assumption (see also Robinson \& Roediger, 1997). However, the existing associative activation account seems incapable of explaining why adding 3 phonological associates to 10 semantic associates (or 3 semantic items to 10 phonological associates) would double false recall unless one assumes 3 items are completely sufficient for either network to reach asymptotic activation. Such an assumption is inconsistent with past research (Hutchison \& Balota, 2005; Robinson \& Roediger, 1997).

A second possibility based on associative activation theory was that over-additive false memory emerged due to interactivity between the semantic and phonological networks. According to Dell's (1986) interactive model of speech production, during vocalisation, interactivity between higher-level semantic concepts and lower-level individual phonemes uniquely isolates the to-be-produced word. As evidence for such interactivity, Dell and O'Seaghdha (1992) demonstrated that speech errors in which the accidentally produced word is both phonologically and semantically similar to the intended target are much more likely than what is predicted based purely on summing the phonological and semantic similarities. For hybrid false memory, such an account suggests that the conceptual context provided by the semantic associates might interact with bottom-up orthographic and phonological information in uniquely isolating the critical lure to surpass a threshold for output.

Although Dell's model dealt with speech production, rather than speech recognition, many speech recognition models, such as the cohort model (Marslen-Wilson \& Tyler, 1980; Marslen-Wilson \& Welsh, 1978), propose interactivity between conceptual context and bottom-up activation. Specifically, within the cohort model, initial phonemes of an auditorily perceived word activate a set of candidates. Moreover, the previous context provided by the speaker's already-spoken words influences which of these activated candidates is selected for recognition, giving priority to context congruent candidates. As discussed by Watson et al. (2003, see also Roediger, Watson, McDermott, \& Gallo, 2001), interactivity across networks could uniquely identify the critical lure. Such convergence could cause critical lures to reach a threshold of conscious awareness during encoding. If so, later retrieval of this conscious experience could cause a reality monitoring failure similar to imagination inflation (e.g., Goff \& Roediger, 1998), in which the person remembers experiencing the critical lure during study but misattributes that experience to having heard it on the study list. This would explain the over-additive effect not just on overall false recall rates, but specifically on false remember responses.

Finally, hybrid lists could make source monitoring more difficult at the time of test, due to confusability between the critical lure and both lexical/semantic and phonological information presented at study. As argued by Watson et al. (2003), participants given pure semantic lists could use the lack of any recollected visual/auditory details that are consistent with the critical lure as diagnostic evidence against its occurrence (see Gallo, 2010). Similarly, given pure phonological lists, they could use the lack of recollected conceptual details relating to the critical lure as evidence against its occurrence (Finley et al., 2017). As a result, an over-additive pattern emerges because source monitoring can be effective in reducing false memories for each of the pure lists, but not for the hybrid list.

\section{Current study}

The current study closely examined the mechanism(s) producing a boost in false recall within hybrid lists. We were particularly interested in testing between Watson et al.'s (2003) second (interactivity of semantic and phonological networks) and third (increased confusability in source monitoring) accounts. To accomplish this, we modelled our experiment after Watson et al.'s (2003) Experiment 1 in which a list of semantic associates is supplemented with additional semantic or phonological items. However, we made three important changes. Our first two changes provided us more precise control over the phonological contributions to false memory above and beyond semantics. For one, we presented 8 semantic associates instead of 12 , after noticing that many items within both Watson et al.'s (2003) semantic lists and traditional Deese/Roediger-McDermott lists also share initial or final phonemes with the critical lure. For instance, the traditional sleep list contains the associates snooze, snore and slumber. Such hybrid items should increase critical lure activation and/ or source confusion even within "pure" lists and would hinder any investigation of the separate effects of semantics and phonology. Reducing the lists to 8 semantic associates allowed us to eliminate any associates from the initial list that shared initial or final phonemes with the critical lure (e.g., snooze and slumber from Watson et al.'s sleep list). Second, we added only a single target item to each list that was either another semantic associate or shared the initial or final phonemes with the critical lure. Both 
Watson et al. (2003) and Finley et al. (2017) found an approximate $10 \%$ boost in false memory from inserting a single phonological item into a list of semantic associates. We therefore anticipated a similar percentage boost in the current experiment. Because the cohort model of speech recognition places special importance on initial phonemes in the activation of potential candidates for conscious identification (Slowiaczek \& Hamburger, 1992), our main predictions concerned the initial phoneme versus the semantic associate; however, we also include the final phoneme target item condition for exploratory purposes because previous hybrid studies do not differentiate between initial versus final phoneme items and because research also demonstrates facilitative effects from rhyming words (Hillinger, 1980).

Our third change was to include not only eight semantic associates related to the critical lure but also eight semantic associates related to a filler DRM lure. This allowed us to present each critical lure's eight semantic associates either before or after our inserted target word while holding the target's list position constant. We did this to test specifically between two accounts of the hybrid boost in false memory: interactive network convergence during encoding versus source monitoring confusion at test. With regard to network convergence, the cohort model of word recognition predicts a boost in false recollection specifically when related associates are presented prior to a target that shares the initial phoneme(s) with the critical lure. This is because the prior context would give priority to the critical lure from among the candidates activated by the target's initial phoneme(s). This, in turn, could cause the critical lure to come to mind during study, leading to later false recollection. By contrast, the source monitoring account would predict equal boosts in false recollection regardless of whether initial or final phonemes match the target and regardless of whether the related associates are presented before or after the target. In all these cases, the target's phonemic overlap with the critical lure should impair participants' ability to later use source monitoring to reject the critical lure.

\section{Method}

\section{Participants}

Eighty four male and female introductory psychology students participated in the study for partial fulfilment of course credit. Previous studies that included a condition with a single inserted phonological item used sample sizes of 56 participants (Watson et al., Experiment 1) and 65-79 participants (Finley et al., Experiment 2). Because we needed a multiple of 6 participants for counterbalancing lists, we chose to run 84 participants in hopes of sufficient power to replicate this effect. All participants were native English speakers. They were run in 60-minute sessions individually or in pairs.

\section{Materials}

Word lists were adapted from 54 of the 55 DRM lists by Roediger et al. (2001). We designated 27 of these lists as critical lists and 27 as filler lists. For all lists, we used the strongest 8 items in backward associative strength (BAS) from list item to critical lure and presented them in descending order. However, individual words were replaced with the next highest BAS item if they contained the same initial or final phonemes as the critical lures. In addition to these 54 Roediger et al. lists, we created six additional lists using the Nelson, McEvoy, and Schreiber (1999) word association norms: three for the critical lists (GOD - bible, church, faith, religion, Jesus, heaven, holy, devil; GUN - holster, pistol, trigger, bullet, rifle, shoot, shot, bang; SICK - ill, flu, nausea, hospital, fever, medicine, well, vomit) and three for the filler lists (HAND - glove, finger, fist, arm, wash, wave, shake, palm; RAIN - umbrella, storm, cloud, hail, puddle, weather, pour, thunder; SNAKE - reptile, rattle, venom, lizard, worm, poison, bite, deadly). The average FAS and BAS was .07 and .29, respectively, for the 30 critical lists and .07 and .12 , respectively, for the 30 filler lists. Finally, for each critical lure (e.g., sleep), we selected three target items: an initial overlap word that contained the same beginning phonemes (e.g., slam), a final overlap word that contained the same ending phonemes (e.g., beep), and an associatively related word that was the 9th or 10th strongest backward associate (e.g., yawn), depending upon whether the 9th highest word was already used to replace a phonologically similar list item earlier in the list. The degree of phonemic overlap with the critical lure was matched across initial overlap targets (1.9 phonemes, range $=1-3$ ) and final overlap targets (1.9 phonemes, range $=1-2$ ) with the goal of overlapping the initial or final two phonemes depending upon condition $[t(29)=0.0, p=1.0$, for the difference in critical lure phonemic overlap across initial and final overlap conditions]. Appendix presents the 30 critical lures and their 3 corresponding target words, along with their corresponding General American standard pronunciation (retrieved from the English Lexicon Project, Balota et al., 2007).

Each of the 30 presented lists contained 17 items: 8 related associates, 8 filler associates (related to a different critical lure) and 1 target word. The target word was always presented in position 9 of the list, with the 8 related associates appearing either before or after the target. Six tapes were created to counterbalance target type (initial overlap, final overlap, or associate) and related order (related associates first vs. last) across participants. Word lists were recorded via cassette tape with a presentation rate of approximately 2 seconds per word.

\section{Design and procedure}

We used a 3 (target condition: initial overlap, final overlap, associate) $\times 2$ (related order: related associates presented before vs. after target item) within-subjects design. Participants seated at a desk were told they would hear a list of 
words and their memory for the words would be tested. After hearing each 17-item word list, participants were immediately given a free recall test along with remember/know instructions (Gardiner, 1988). On the recall test, participants were instructed to write down as many words as they could remember. Also, each blank line on the recall test contained the letters $\mathrm{R}$ and $\mathrm{K}$ at the end. Participants were informed to circle $\mathrm{R}$ for remember, if they "distinctly remember having heard the item. In other words, you have a vivid memory of the actual presentation". They were further told that:

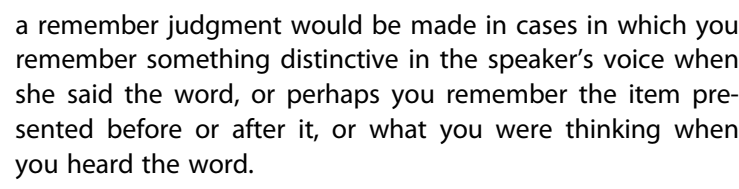

They were told to circle K for know if they "know the item was in the previous list, but cannot remember specific details about its presentation and lack the feeling of remembering the actual occurrence of the word". They repeated this study-test task for all 30 lists within a 60minute time period.

\section{Results}

For critical lures, list items, and target items, we separately examined false recall, remember responses, and know responses as a function of inserted target condition and related order. In addition to the lures and list items from the critical lists, we also analysed the lures and list items from filler lists. Condition Means and standard errors for each type of item are shown in Table 1 for critical lists and Table 2 for filler lists. All reported significant effects are associated with a two-tailed $p$-value of .05 .

\section{Critical lures}

\section{Critical lists}

For overall false recall, remember and know responses, there was a main effect of related order, such that participants showed higher false recall $[F(1,83)=19.99, \mathrm{MSE}=$ $\left.311, \eta_{p}^{2}=.19\right]$, more remember responses $[F(1,83)=4.68$, $\left.p=.03, \eta_{p}^{2}=.05\right]$, and more know responses $[F(1,83)=$ 9.99, $\left.\mathrm{MSE}=248, \eta_{p}^{2}=.11\right]$ to critical lures when their corresponding 8 semantic associates were presented in the first half of the list. Also, and as predicted, the two-way interaction between target condition and related order was significant for remember responses, $F(2,82)=3.70, \mathrm{MSE}=155$, $\eta_{p}^{2}=.04$. Follow-up comparisons revealed that, when related list items were presented first, inserting an initial overlap target boosted remember responses by $6.6 \pm 3.8 \%$ relative to inserting an associate target $[t=95 \%$ confidence interval]. However, when related list items were presented after the target item, inserting an initial overlap target did not influence remember responses relative to the associate target $[-0.7 \pm 3.8 \%$, see Table 1]. The effect of inserting final overlap targets was more equivocal; remember
Table 1. Proportion recall, remember, and know responses for critical lures (top), list items, (middle) and target items (bottom) as a function of inserted target item and related order. Standard errors are in parentheses.

\begin{tabular}{|c|c|c|c|}
\hline Target item & Recall & Remember & Know \\
\hline \multicolumn{4}{|c|}{ Critical lures } \\
\hline \multicolumn{4}{|c|}{ Related associates first } \\
\hline Initial overlap & $.26(.02)$ & $.14(.02)$ & $.12(.02)$ \\
\hline Final overlap & $.23(.02)$ & $.11(.02)$ & $.11(.02)$ \\
\hline Associate & $.19(.02)$ & $.07(.01)$ & $.12(.02)$ \\
\hline \multicolumn{4}{|c|}{ Related associates second } \\
\hline Initial overlap & $.15(.02)$ & $.07(.02)$ & $.08(.01)$ \\
\hline Final overlap & $.17(.02)$ & $.10(.02)$ & $.08(.01)$ \\
\hline Associate & $.14(.02)$ & $.08(.01)$ & $.06(.01)$ \\
\hline \multicolumn{4}{|c|}{ List items } \\
\hline \multicolumn{4}{|c|}{ Related associates first } \\
\hline Initial overlap & $.55(.02)$ & $.48(.02)$ & $.07(.01)$ \\
\hline Final overlap & $.54(.02)$ & $.46(.02)$ & $.07(.01)$ \\
\hline Associate & $.54(.02)$ & $.46(.02)$ & $.08(.01)$ \\
\hline \multicolumn{4}{|c|}{ Related associates second } \\
\hline Initial overlap & $.60(.01)$ & $.51(.02)$ & $.08(.01)$ \\
\hline Final overlap & $.58(.01)$ & $.50(.02)$ & $.08(.01)$ \\
\hline Associate & $.59(.01)$ & $.51(.02)$ & $.08(.01)$ \\
\hline \multicolumn{4}{|c|}{ Target items } \\
\hline \multicolumn{4}{|c|}{ Related associates first } \\
\hline Initial overlap & $.26(.03)$ & $.22(.03)$ & $.04(.01)$ \\
\hline Final overlap & $.22(.02)$ & $.19(.02)$ & $.03(.01)$ \\
\hline Associate & $.30(.03)$ & $.25(.02)$ & $.05(.01)$ \\
\hline \multicolumn{4}{|c|}{ Related associates second } \\
\hline Initial overlap & $.21(.03)$ & $.16(.02)$ & $.04(.01)$ \\
\hline Final overlap & $.26(.02)$ & $.22(.02)$ & $.04(.01)$ \\
\hline Associate & $.35(.03)$ & $.30(.03)$ & $.05(.01)$ \\
\hline
\end{tabular}

responses for final overlap targets did not significantly differ from remember responses for associate targets or initial overlap targets regardless of whether related items were presented before the target [differences of $4.0 \pm$ $4.1 \%$ and $-2.5 \pm 3.8 \%$, respectively] or after the target [differences of $1.4 \pm 4.1 \%$ and $2.1 \pm 4.5 \%$, respectively]. The two-way interaction between target condition and related order remained significant for remember responses after eliminating the exploratory final overlap condition, as predicted $F(1,83)=9.24, \mathrm{MSE}=121, \eta_{p}^{2}=.10$. There was no overall effect of target condition $\left[p=.89, \eta_{p}^{2} s=.00\right]$ and the related order $\times$ target condition interaction did not

Table 2. Proportion recall, remember and know responses for filler lures (top) and their associated filler list items as a function of inserted target item and related order. Standard errors are in parentheses.

\begin{tabular}{lccc}
\hline Target item & Recall & Remember & Know \\
\hline & Filler lures & & \\
Filler associates first & & & \\
$\quad$ Initial overlap & $.08(.01)$ & $.06(.01)$ & $.02(.01)$ \\
$\quad$ Final overlap & $.07(.01)$ & $.05(.01)$ & $.02(.01)$ \\
$\quad$ Associate & $.06(.01)$ & $.03(.01)$ & $.03(.01)$ \\
Filler associates second & & & \\
$\quad$ Initial overlap & $.06(.01)$ & $.02(.01)$ & $.03(.01)$ \\
Final overlap & $.05(.01)$ & $.02(.01)$ & $.04(.01)$ \\
Associate & $.05(.01)$ & $.02(.01)$ & $.03(.01)$ \\
& Filler list items & & \\
Filler associates first & & & $.07(.01)$ \\
$\quad$ Initial overlap & $.52(.02)$ & $.45(.02)$ & $.08(.01)$ \\
$\quad$ Final overlap & $.51(.02)$ & $.44(.02)$ & $.07(.01)$ \\
$\quad$ Associate & $.51(.02)$ & $.44(.02)$ & \\
Filler associates second & & & $.09(.01)$ \\
$\quad$ Initial overlap & $.55(.02)$ & $.47(.02)$ & $.09(.01)$ \\
$\quad$ Final overlap & $.58(.02)$ & $.49(.02)$ & $.07(.01)$ \\
$\quad$ Associate & $.58(.02)$ & $.51(.02)$ & \\
\hline
\end{tabular}


approach significance $\left[p=.54, \eta_{p}^{2} s=.01\right]$ for know responses.

Surprisingly, the related order $\times$ target item interaction was not significant for overall recall $F(2,166)=1.12, \mathrm{MSE}=$ $299 \eta_{p}^{2}=.01$. Such an interaction would be expected if inserting an initial overlap target simply boosted remember responses, while leaving know responses unaffected. In fact, numerically this is the case. Inspection of Table 1 shows the same pattern for overall false recall as for the remember data, with initial overlap targets producing an increase in false recall only when presented after the semantic associates. Pairwise comparisons confirmed this pattern, such that the $7 \%$ increase for false recall was significant when the critical semantic associates preceded the target $[t(83)=2.37, p=.02]$, but not when the critical semantic associates occurred after the target $[t(83)=$ $0.53, p=.60]$. The significant effect for false recall following semantic associates replicates previous hybrid false recall studies, because all previous studies have also positioned the phonological item following at least some of the semantic associates. ${ }^{1}$ Finally, when the exploratory final overlap condition is excluded from the analysis, the recall order $\times$ target condition interaction approaches significance for overall false recall, $F(1,83)=3.00, \mathrm{MSE}=193$, $p=.09, \eta_{p}^{2}=.04$. Thus, we are confident that the overall false recall data both replicate previous hybrid false recall studies and reflect the same pattern as our remember response data.

\section{Filler lists}

Overall false recall was quite low for filler lists ( $M=6 \%$, see Table 2). There was an order effect in overall false recall and remember responses that mimicked the pattern for the critical lists. Specifically, participants showed higher false recall $\left[F(1,83)=4.61, \mathrm{MSE}=101, \eta_{p}^{2}=.05\right]$ and more remember responses $\left[F(1,83)=12.13, \mathrm{MSE}=69, \eta_{p}^{2}=.13\right]$ when the filler lure's semantic associates were presented in the initial half of the list. No other effects approached significance.

\section{List items}

\section{Critical lists}

For list item overall recall and remember responses, there was a main effect of related order $[F(1,83)=16.83$, MSE $=164, \eta_{p}^{2}=.17 ; F(1,83)=7.03, \mathrm{MSE}=262, \eta_{p}^{2}=.08$ for recall and remember responses, respectively] such that the critical semantic associates were $4.7 \pm 2.2 \%$ more likely to be recalled and $3.8 \pm 2.9 \%$ more likely to be remembered when presented in the second half of the list than when presented in the beginning of the list. Neither the main effect of target condition [both $p$-values $\left.>.19, \eta_{p}^{2} s<.02\right]$ nor the related order $\times$ target condition interaction [both $p$-values $>.68, \eta_{p}^{2} s<.01$ ] approached significance. For know responses, none of the effects approached significance (all $F_{s}<1, p s>.38, \eta_{p}^{2} s<.01$ ).

\section{Filler lists}

When we analysed list item recall from filler lists, we found an effect of related order that was significant in recall and remember responses $\left[F(1,87)=20.47, \mathrm{MSE}=207, \eta_{p}^{2}=.19\right.$ and $F(1,87)=11.72, \mathrm{MSE}=274, \eta_{p}^{2}=.12$, respectively] and marginal for know responses, $F(1,87)=3.13$, MSE $=$ $50, \eta_{p}^{2}=.04$ (see Table 2). As was found for list items from the critical lists, the filler list items were $5.7 \pm 2.5 \%$ more likely to be recalled and $4.9 \pm 2.8 \%$ more likely to be remembered when presented in the second half of the list than when presented in the beginning of the list. In addition to this main effect, there was a target condition $\times$ related order interaction that was significant for remember responses $\left[F(2,174)=3.10, \mathrm{MSE}=84, \eta_{p}^{2}=.19\right]$ and marginal for overall recall, $F(2,174)=2.75, \mathrm{MSE}=64, \eta_{p}^{2}=.03$. Separate pairwise comparisons for each related order revealed that there were no differences in remember responses across target conditions when the filler associates were presented first (all $p s>.40$ ); however, when the filler associates were presented after the target item, they were more likely to be remembered following a semantic associate target than an initial overlap target (a $3.6 \pm$ $2.9 \%$ difference) or an end overlap target (a marginal $2.2 \pm 2.3 \%$ difference).

\section{Target items}

For target items overall recall and remember responses, the effect of related order was not significant $(F<1)$, but there was a significant main effect of target condition, $F(2,166)=11.37, \mathrm{MSE}=357, \eta_{p}^{2}=.12 ; F(2,166)=10.36$, $\mathrm{MSE}=330, \eta_{p}^{2}=.11$ for recall and remember responses, respectively. Planned comparisons revealed that associate targets were recalled $8.7 \pm 4.5 \%$ and recollected $8.2 \pm 4.3 \%$ more frequently than initial overlap targets and recalled $8.3 \pm 4.2 \%$ and recollected $7.3 \pm 3.9 \%$ more frequently than final overlap targets. The initial overlap and final overlap targets did not differ in either recall $[-0.4 \pm$ $3.6 \%]$ or remember responses [ $-1.0 \pm 3.6 \%]$. Additionally, the interaction between related order and target condition was significant for both recall and remember responses $\left[F(2,166)=4.86, \mathrm{MSE}=282, \eta_{p}^{2}=.06 ; F(2,166)=6.10\right.$, $\mathrm{MSE}=231, \eta_{p}^{2}=.07$ for recall and remember responses, respectively]. To decompose this interaction, we used pairwise comparisons to examine the effect of related order separately for each target item. We found that recall and remember responses of initial overlap targets significantly differed across related order, with initial overlap targets recalled $5.2 \pm 5.1 \%$ and recollected $5.7 \pm 5.6 \%$ more often when the critical list semantic associates were presented prior to, rather than after, the target. In contrast, recall and remember responses for associate targets were marginally greater $(5.5 \pm 5.9 \%$ and $5.1 \pm$ $5.5 \%$, respectively) when critical list semantic associates were presented after the target and performance for final overlap targets did not differ (both $p s>.11$ ). There 
were no significant effects on know responses $(F s<1$, ps $\left.>.63, \eta_{p}^{2} s<.01\right)$.

\section{Discussion}

The current results provide evidence that the boost in false memory from hybrid lists is due to interactivity between semantic and phonological networks during encoding. As an example of such an interactive model, the cohort model of word recognition predicts that inserting a target item with similar initial phonemes as the critical lure should activate a set of candidates that includes the critical lure. In addition, this model further predicts that preceding the inserted target with associated list items should increase top-down selection of the critical lure from among other candidates (perhaps causing it to reach a threshold for consciousness). This should, in turn, increase remember responses of critical lures only under the specific condition in which an initial overlap target is inserted after (but not before) associated list items. Our results confirmed this very specific prediction from the cohort model. Specifically, inserting an initial overlap target increased experiences of false recollection of the critical lure, but only when words associated to the critical lure were presented prior to, rather than after, the inserted target.

In contrast, the other previous explanations for hybrid false memory cannot capture the current data. For instance, the associative activation account predicts that false memory is a result of converging activation from phonological and semantic networks that is over-additive relative to saturation that occurs within either network alone when additional associates are encountered (Finley et al., 2017; Watson et al., 2003). However, this account cannot explain why the hybrid "boost" in false remembering occurs only when the phonological associate was presented following semantic associates, but not before. Similarly, the source monitoring account predicts an increase in false remember responses of critical lures whenever a phonologically similar item is inserted, regardless of related order, because this potentially recollected phonological information could provide misleading diagnostic evidence for the critical lure's presentation during study. As with the converging network activation account, the finding of a boost in false remembering only when related associates occur first is inconsistent with such a general source monitoring account.

In addition to the failures of simple associative activation and source monitoring accounts, fuzzy trace theory (Brainerd \& Reyna, 1990) is also unable to explain the current data. Fuzzy trace theory accounts for false memory through a strong "gist" trace of semantic content encoded during study. Because this trace is based on semantics, it cannot currently explain phonological-based false memory or hybrid "boosts" in false memory (Sommers \& Lewis, 1999; Finley et al., 2017). One could, perhaps, extend fuzzy trace to assume we extract "phonological gists" from lists with many phonological associates (e.g., the "dog" list of log, dodge, dug, hog, bog, dock, etc
...). However, when dealing with only a single phonological target, it is not clear how a gist trace would differ from a verbatim trace of that phonology. Alternatively, perhaps people could use the retrieved verbatim trace of the target item (including its phonology) in combination with the semantic gist extracted from the semantic associates to infer the critical lure's presentation. However, such an account could not explain the critical interaction with related order or why the false memory boost appeared in remember responses, rather than know responses.

We believe the most complete account of our data combines source misattribution with activation using the cohort model to guide assumptions of speech recognition during encoding. Specifically, combining top-down context and bottom-up candidate activation specifically isolates the critical lure, causing it to come to mind during encoding. During recall, participants could then misattribute their internal (perhaps subvocal) identification of the critical lure during study as having actually heard it presented. Such an account nicely integrates memory processes with word recognition processes, which will always play a role when participants study word lists.

One notable aspect of the study is the relatively small size of the hybrid boost to false memory, relative to past hybrid false memory studies. The main reason for this is that we wanted to specifically isolate the benefit of inserting a single phonological item, which reduces the effect size relative to lists with more hybridisation (Finley et al., 2017). Although we were able to do so, this could reduce our ability to generalise our results to studies with more hybrid lists. Still, our 7\% boost in false memory and false "remember" responses from an initial overlap phonological item was slightly less than the $10 \%$ boost seen in previous studies. One possible reason for this is that our lists contained 8 related associates, whereas previous studies included lists with 15 semantic associates. Lists with 15 associates could more strongly strengthen the lure's semantic representation to asymptotic levels, potentially allowing for a larger boost from an inserted phonological target, relative to yet another semantic associate. In terms of generalisability to other studies, because we were particularly interested in testing a prediction from the cohort model, we chose to present our lists auditorily. It is therefore unclear whether these results would generalise to the more common visual presentation of lists. Similarly, because most hybrid false memory studies have used recall (but see Watson et al., Experiment 3) we chose to also examine free recall and inserted remember/know judgments within the free recall task. However, remember/know judgments are more typically examined in recognition tests and future studies should investigate whether these patterns would replicate in recognition.

\section{Other findings}

Although this study was concerned primarily with the potential effects of target condition and interaction 
with order of associate presentation, there were three additional findings obtained. First, there were dissociative main effects of related order on critical lure and list item recall. Specifically, list item recall was greatest for items in the second half of the list, whereas critical lure recall was greatest when the associates related to the critical lure were presented in the first half of the list. We do not know of any other studies showing such a main effect for critical lure recall. However, past studies have shown that list item recall is negatively associated with critical lure recall (Roediger et al., 2001), so it is likely that greater recall for items presented in the second half of the list led to reduced false memory from these items. This might be especially pronounced with 8-item associate lists, in which participants might have an easier time using the absence of remembered perceptual details to dismiss a critical item's presentation in the presence of distinct item recollection for the other 8 items presented in the second half of the list. Second, filler list items that followed the inserted target were remembered better if the target was a semantic associate of the critical list lure than if it was an initial or final overlap item of the critical lure. Although speculative, we believe this might be due to the two phonological overlap targets, once identified, serving to break up the semantic context established by the critical semantic associates prior to presentation of the filler list. In contrast, if the inserted target word is yet another semantic associate, then the filler list itself serves to break up the previous context and start a new theme. This change in context and immediate instantiation of a new semantic context can cause greater attention and relational processing among filler list items. Third, semantic associate target items were recalled more often than the initial or final overlap words. This is unsurprising because these items fit the semantic theme of the list and would have benefitted from relational processing of the other associated items in the list.

\section{Conclusion}

This experiment provided a crucial test examining the source of the hybrid boost in false recall. Our findings were inconsistent with the source monitoring account and also inconsistent with an account based on simple additive or interactive activation across semantic and phonological networks. Furthermore, the existence of a hybrid boost in false recall is also inconsistent with the fuzzy trace model (Brainerd \& Reyna, 2002) which currently has no mechanism for phonological contributions to false memory. We believe the most accurate explanation is a modification of the activation-monitoring theory that incorporates the interactivity between top down semantic context and bottom-up word activation during encoding combined with source monitoring failure at retrieval.

\section{Note}

1. The only exception is Finley et al. (2017) in which the position of each list item was randomly selected or counterbalanced across subjects and one of the lists contained 15 semantic associates and 1 phonological associate. However, because there were 16 items per list, this phonological item still would have been preceded by at least 1 semantic item $94 \%$ of the time $(15 / 16)$.

\section{Acknowledgements}

Special thanks to Gabriel Abate, Ben Baldassare, Kelly Buchanan, Bethany Johannessen and Reema Najjar for scheduling and running participants for this study.

\section{Disclosure statement}

No potential conflict of interest was reported by the authors.

\section{References}

Balota, D. A., Yap, M. J., Cortese, M. J., Hutchison, K. A., Kessler, B., Loftus, B., ... Treiman, R. (2007). The English lexicon project: A user's guide. Behavior Research Methods, 39, 445-459.

Brainerd, C. J., \& Reyna, V. F. (1990). Gist is the grist: Fuzzy-trace theory and the new intuitionism. Developmental Review, 10, 3-47.

Brainerd, C. J., \& Reyna, V. F. (2002). Fuzzy-trace theory and false memory. Current Directions in Psychological Science, 11, 164-169.

Deese, J. (1959). On the prediction of occurrence of particular verbal intrusions in immediate recall. Journal of Experimental Psychology, $58,17-22$.

Dell, G. S. (1986). A spreading-activation theory of retrieval in sentence production. Psychological Review, 93, 283-321.

Dell, G. S., \& O'Seaghdha, P. G. (1992). Stages of lexical access in language production. Cognition, 42, 287-314.

Finley, J. R., Sungkhasettee, V. W., Roediger, H. L. III, \& Balota, D. A. (2017). Relative contributions of semantic and phonological associates to over-additive false recall in hybrid DRM lists. Journal of Memory and Language, 93, 154-168.

Gallo, D. A. (2006). Associative illusions of memory. New York, NY: Psychology Press.

Gallo, D. A. (2010). False memories and fantastic beliefs: 15 years of the DRM illusion. Memory \& Cognition, 38, 833-848.

Gardiner, J. M. (1988). Functional aspects of recollective experience. Memory \& Cognition, 16, 309-313.

Goff, L. M., \& Roediger, H. L., III. (1998). Imagination inflation for action events: Repeated imaginings lead to illusory recollections. Memory \& Cognition, 26, 20-33.

Hillinger, M. L. (1980). Priming effects with phonemically similar words: The encoding-bias hypothesis reconsidered. Memory \& Cognition, 8 , 115-123.

Hutchison, K. A., \& Balota, D. A. (2005). Decoupling semantic and associative information in false memories: Explorations with semantically ambiguous and unambiguous critical lures. Journal of Memory and Language, 52, 1-28.

Marslen-Wilson, W. D., \& Tyler, L. K. (1980). The temporal structure of spoken language understanding. Cognition, 8, 1-71.

Marslen-Wilson, W. D., \& Welsh, A. (1978). Processing interactions and lexical access during word-recognition in continuous speech. Cognitive Psychology, 10, 29-63.

Nelson, D. L., McEvoy, C. L., \& Schreiber, T. (1999). The University of South Florida Word association, rhyme and word fragment norms. http://www.usf.edu/FreeAssociation/

Payne, D. G., Elie, C. J., Blackwell, J. M., \& Neuschatz, J. S. (1996). Memory illusions: Recalling, recognizing, and recollecting events that never occurred. Journal of Memory \& Language, 35, 261-285. 
Robinson, K. J., \& Roediger, H. L. (1997). Associative processes in false recall and false recognition. Psychological Science, 8, 231-237.

Roediger, H. L., Balota, D. A., \& Watson, J. M. (2001). Spreading activation and the arousal of false memories. In $\mathrm{H}$. L. Roediger, J. S. Naire, I. Neath, \& A. M. Suprenant (Eds.), The nature of remembering: Essays in honor of Robert G. Crowder (pp. 95-115). Washington, DC: American Psychological Association Press.

Roediger, H. L., \& McDermott, K. B. (1995). Creating false memories: Remembering words not presented in lists. Journal of Experimental Psychology: Learning, Memory, and Cognition, 21, 803-814.

Roediger, H. L., Watson, J. M., McDermott, K. B., \& Gallo, D. A. (2001). Factors that determine false recall: A multiple regression analysis. Psychonomic Bulletin \& Review, 8, 385-407.

Slowiaczek, L. M., \& Hamburger, M. B. (1992). Prelexical facilitation and lexical interference in auditory word recognition. Journal of Experimental Psychology: Learning, Memory, and Cognition, 18, 1239-1250.
Sommers, M. S., \& Lewis, B. P. (1999). Who really lives next door: Creating false memories with phonological neighbors. Journal of Memory and Language, 66, 376-383.

Tse, C. S., Li, Y., \& Neill, W. T. (2011). Dlssociative effects of phonological vs. semantic associates on recognition memory in the Deese/Roediger-McDermott paradigm. Acta Psychologia, 137, 269-279.

Underwood, B. J. (1965). False recognition produced by implicit verbal responses. Journal of Experimental Psychology, 70, 122-129.

Watson, J. M., Balota, D. A., \& Roediger III, H. L. (2003). Creating false memories with hybrid lists of semantic and phonological associates: Over-additive false memories produced by converging associative networks. Journal of Memory \& Language, 49, 95-118.

Watson, J. M., Balota, D. A., \& Sergent-Marshall, S. D. (2001). Semantic, phonological, and hybrid veridical and false memories in healthy older adults and in individuals with dementia of the Alzheimer type. Neuropsychology, 15, 254-267.

\section{Appendix}

Table A1. Thirty critical lures and their 3 corresponding target words, with General American standard pronunciation in parentheses generated from the English Lexicon Project (Balota et al., 2007).

\begin{tabular}{|c|c|c|c|c|c|c|c|}
\hline \multicolumn{2}{|c|}{ Critical Lure } & \multicolumn{2}{|c|}{ Initial Overlap } & \multicolumn{2}{|c|}{ Final Overlap } & \multicolumn{2}{|c|}{ Associate } \\
\hline anger & ("aN.g@') & angle & ("aN.gl=) & ogre & ("o.g@") & fight & (f"alt) \\
\hline black & (bl"ak) & blend & (bl"End) & crack & (kr"ak) & grief & (gr"if) \\
\hline car & $\left(\mathrm{k}^{\prime \prime} \mathrm{Ar}\right)$ & cool & (k"ul) & bar & (b"Ar) & jeep & (dZ"ip) \\
\hline chair & (tS"Er) & choke & (tS"ok) & mare & (m"Er) & sofa & (s"o.f@) \\
\hline city & $\left(s^{\prime \prime} \mid .4 i\right)$ & simple & (s"Im.pl=) & party & (p"Ar.4i) & streets & (str"its) \\
\hline cold & (k"old) & comb & (k"om) & build & (b"lld) & winter & (w"In.4@') \\
\hline cup & (k"Vp) & come & (k"Vm) & pup & $\left(p^{\prime \prime} \vee p\right)$ & stein & (st"aln) \\
\hline flag & (fl"ag) & flick & $\left(\mathrm{fl} \mathrm{l}^{\prime \prime} \mathrm{k}\right)$ & stag & (st"ag) & symbol & $\left(s^{\prime \prime} \mid \mathrm{m} \cdot \mathrm{bl}=\right)$ \\
\hline foot & $\left(f^{\prime \prime} U t\right)$ & full & (f"UI) & soot & $\left(s^{\prime \prime} U t\right)$ & knee & $\left(n^{\prime \prime i}\right)$ \\
\hline girl & $\left(g^{\prime \prime} 3^{\prime} l\right)$ & girth & (g"3’T) & pearl & $\left(p^{\prime \prime} 3^{\prime} l\right)$ & niece & (n"is) \\
\hline god & (g"Ad) & gauze & (g"Oz) & pod & (p"Ad) & bless & (bl"Es) \\
\hline gun & (g"Vn) & gut & $\left(g^{\prime \prime} \mathrm{Vt}\right)$ & fun & $\left(f^{\prime \prime} \mathrm{Vn}\right)$ & hunt & (h"Vnt) \\
\hline mountain & (m"aUn.tn=) & mouthful & (m"aUT.fUI) & pardon & $\left(p^{\prime \prime} A r \cdot d n=\right)$ & bike & (b"alk) \\
\hline music & (mj"u.zlk) & muted & (mj"u4.@d) & public & (p"V.bllk) & instrument & ("In.str@.m@nt) \\
\hline needle & $\left(n^{\prime \prime} i .4 \mid=\right)$ & kneeling (n"il.IN) & pedal & $\left(p^{\prime \prime} E .4 \mid=\right)$ & sharp & (S"Arp) & \\
\hline river & (r"I.v@) & risky & (r"Isk.i) & over & ("o.v@") & boat & (b"ot) \\
\hline rough & $\left(r^{\prime \prime} \mathrm{Vf}\right)$ & runt & (r"Vnt) & bluff & (bl"Vf) & ground & (gr"aUnd) \\
\hline rubber & (r"Vb.@') & rustle & $\left(r^{\prime \prime} \mathrm{V} . \mathrm{sl}=\right)$ & member & (m”Em.b@) & glue & (gl"u) \\
\hline sick & $\left(s^{\prime \prime} \mid k\right)$ & silt & $\left(s^{\prime \prime} \mid l t\right)$ & pick & $\left(p^{\prime \prime} \mid k\right)$ & cough & (k"Of) \\
\hline sleep & (sl"ip) & slam & (sl"am) & beep & (b"ip) & yawn & (j"On) \\
\hline slow & (sl"o) & slid & $\left(\left.s\right|^{\prime \prime} \mid d\right)$ & row & $\left(r^{\prime \prime} 0\right)$ & traffic & (tr"a.flk) \\
\hline smell & (sm"El) & smack & (sm"ak) & fell & (f"El) & hear & (h"lr) \\
\hline smoke & (sm"ok) & smart & (sm"Art) & poke & (p"ok) & fire & (f"alr) \\
\hline soft & (s"Oft) & sauce & (s"Os) & lift & $\left(I^{\prime \prime} \mid \mathrm{ft}\right)$ & skin & (sk"ln) \\
\hline spider & (sp"al.4@") & spastic & (sp"a.stlk) & under & ("Vn.4@”) & crawl & (kr"Ol) \\
\hline sweet & (sw"it) & swoon & (sw"un) & meat & $\left(m^{\prime \prime} i t\right)$ & soda & (s"o.4@) \\
\hline trash & (tr"aS) & trail & (tr"el) & splash & (spl"aS) & pile & (p"all) \\
\hline window & (w"In.do) & whisper & (hw"l.sp@') & meadow & (m"E.do) & shade & (S"ed) \\
\hline
\end{tabular}

